\title{
Interactive comment on "EBSD in Antarctic and Greenland Ice" by Ilka Weikusat et al.
}

\section{Anonymous Referee \#1}

Received and published: 18 March 2017

The manuscript "EBSD in Antarctic and Greenland Ice" submitted by Weikusat et al. for publication in SE represents a valuable contribution towards a better understanding of the flow behavior of polar ice sheets. The flow behavior of ice is an important topic in view of all glacial and sub-glacial Earth surface processes. The detailed and sophisticated light microscopic and EBSD data are well documented and the interpretations are comprehensive.

General comments are listed as follows:

1. The main observation of the study is a high amount of subgrain boundaries made up of dislocations representing non-basal glide systems. This observation is not entirely new but the study confirms their common occurrence in natural polar ice sheets.

Yet, the implication that this will "have a major impact on the discussion of strain-rate controlling processes" (abstract line 15, conclusions, page 15, lines 6-7) seems to be 
overemphasized, as suggested by their discussion on page 14, lines 4-17: Even a minor activity of non-basal glide produces a high amount of relative immobile dislocations that are arranged into low angle grain boundaries. Thus, low angle grain boundaries made up of dislocations gliding on non-basal planes do not represent rate-controlling glide systems.

2. The glide system responsible for the main strain can most probably be best inferred from the CPO, yet the CPO is not documented in this study. It would be helpful to describe it, although it is not strongly developed, as stated on page 5, lines 22-24.

3. Generally, some more observations and discussions indicating that indeed deformation by dislocation glide is the main deformation mechanism of the studied ice samples would be helpful.

Specific comments are listed as follows:

- Page 1, line 15, rephrase, see general comment 1.

- Page 1 lines 16/17: "host grain alternative formation processes" please specify, which are these alternative formation processes (e.g., alternative to what..., what are the alternatives...).

- Page 2, line 12: "various deformation mechanisms": please specify the various deformation mechanisms

- Page 2, line 15, please specify: what is the main evidence for the interpretation that dislocation glide is the main deformation mechanism

- Page 3, line 22 (and throughout the text): please add: low misorientation "angles" < $5^{\circ} \ldots$

- Page 3, line 25: please rephrase, e.g. ... "the orientation/azimuth of" the c-axis of the ice is known....

- Page 5, lines 22-25: Please add some information on the CPO, as this is important 
to judge the importance of dislocation glide as main deformation mechanism

- Page 12, line 10 and following: please rearrange the listing of the seven types of SED subgrain boundaries recognized by Means and Ree, 1988. For example, give in the beginning of this discussion a short overview what are the main differences.

- Page 15, line 6, rephrase, see general comment 1.

Interactive

comment

- Page 5, line 11: strong CPO? Not in your sample?

References - Missing references in the list: Means and Ree, 1988; Mainprice et al., 1933

- Reference list: the year is at various positions in the listed references

- Please also cite the experimental work on the deformation behavior of ice by Piazolo et al., 2013; Cyprych et al., 2016

Figures

- Fig. 1: What would be the appearance of cleavage fractures in ice? Figure 1a) illustrates the "p-type sgb swarm". Given the low misorientation angle involved with these planar microstructures, could they represent cleavage fractures?

Interactive comment on Solid Earth Discuss., doi:10.5194/se-2017-12, 2017. 